\title{
La médecine de pointe en Suisse
}

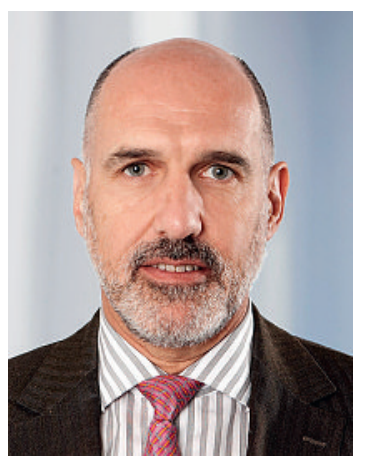

La FMH s'est peu préoccupée, jusqu'à présent, de la médecine hautement spécialisée en Suisse, de la «médecine de pointe», comme on dit.

On peut se demander pourquoi, alors que cela nous concerne au premier chef, préoccupés que nous sommes tant de la médecine que de notre système de santé. Peutêtre est-ce parce que c'est là une question à fort potentiel émotionnel, qui divise, qui réveille à tout coup des tensions qu'on préférerait enfouies laissons d'autres se brûler les doigts! Mais c'est aussi probablement parce que le corps médical est soigneusement laissé hors des discussions à ce sujet, comme si tout était déjà assez compliqué sans qu'on y implique encore les principaux intéressés...

Nous avons donc décidé d'affronter cette question beaucoup plus activement. Nous y consacrerons la traditionnelle rencontre de la FMH avec les parlementaires fédéraux, qui a lieu à chaque début de session, et publions simultanément à ce sujet un "papier de position» [1].

\section{Ce qu'il faut d'abord à celles et ceux qui en appellent à la médecine hautement spécialisée, ce sont des prestations de la meilleure qualité}

La médecine hautement spécialisée est au croisement de nombreux axes de réflexion très actuels: fédéralisme, coûts du système de santé, accès aux soins, optimisation de la qualité, innovation et financement de cette dernière, c'est un vrai bouquet de questions chaudes qui se noue ici.

L'essentiel est cependant de ne pas perdre de vue l'intérêt des patientes et des patients qui ont besoin de ces prestations de pointe. La prise en compte de l'intérêt des malades permet de hiérarchiser ces divers éléments de réflexion, et simultanément, on le verra, de garantir aussi les intérêts du corps médical.

Ce qu'il faut d'abord à celles et ceux qui en appellent à la médecine hautement spécialisée, ce sont des prestations de la meilleure qualité; et cela signifie forcément regroupement, on le sait: l'expérience des équipes soignantes, leur habitude de travailler ensemble, c'est-à-dire le nombre de cas auxquels elles sont confrontées chaque année, tout cela est essentiel. Au vu des expériences faites à l'étranger, selon les domaines, au-delà d'un ou deux centres en Suisse, on n'atteindra pas la masse critique nécessaire.

Ce qu'il faut ensuite aux patient-es, c'est évidemment d'avoir accès aux prestations nécessaires. Géographiquement, c'est clair, mais on peut certainement demander à un patient de se déplacer de 100 ou $200 \mathrm{~km}$ pour une intervention «de pointe», et ce problème n'en est en fait pas un si l'on s'assure que la barrière linguistique pourra être surmontée. Mais aussi au niveau financier et administratif, dans le sens où les prestations hautement spécialisées, si elles font sens, doivent être reconnues par les structures tarifaires, y compris les innovations et les opérations complexes.

Et qu'en est-il maintenant des médecins? Je l'ai dit, on retrouve les mêmes besoins, les mêmes exigences que pour les patient-es; ce n'est pas étonnant, mais surtout cela ne peut que renforcer notre motivation à œuvrer à des solutions efficaces et rapides. Pour les médecins aussi, qualité et innovation sont les éléments centraux de la médecine hautement spécialisée que nous souhaitons pour notre pays.

Un regroupement radical des activités en médecine hautement spécialisée est pour les médecins un facteur de qualité incontournable; et l'existence de centres vraiment performants et reconnus est un facteur de motivation pour l'ensemble de la profession comme dans l'optique de la relève; la compétitivité de notre pays dans ce domaine est aussi un élément essentiel dans le recrutement des spécialistes nécessaires, et dans la constitution d'équipes de pointe. Le regroupement des centres de médecine hautement spécialisée est donc pour la FMH une exigence urgente.

Et pour nous aussi, comme pour les patient-es, l'innovation doit être à la fois soutenue et reconnue sur le plan tarifaire. Le financement des innovations doit donc absolument être réglé dans le cadre de la structure DRG.

Bref, la médecine hautement spécialisée est actuellement encore beaucoup trop marquée, en Suisse, par les particularités locales et par une vision fragmentée des intérêts économiques et politiques. Pour en assurer la qualité, le développement et la compétitivité, le regroupement de la médecine «de pointe» est une urgence aux yeux du corps médical, et la FMH a décidé de s'investir davantage dans ce problème, pour contribuer à des solutions d'avenir.

Dr Jacques de Haller, Président de la FMH

1 www.fmh.ch $\rightarrow$ Politique \& Médias $\rightarrow$ Prises de position de la FMH 